\title{
Voltammetric Tracing of Al(III) Using Supramolecular Metal-Polyphenolic Nanofilms Obtained via Electrochemically Assisted Self-Assembly
}

\author{
A. Krywko-Cendrowska ${ }^{\S^{*}}$ \\ §SCS-DSM Award for best poster presentation in Polymers, Colloids \& Interfaces
}

\begin{abstract}
Supramolecular metal-polyphenolic thin sensor films represent a unique class of composite materials. Their properties and sensitivity can be easily modified via controlled self-assembly of their molecular components. Among the different assembly methods, electrochemically triggered processes are extremely powerful because they allow spatial confinement of the film buildup via an electrical stimuli-controlled process. In this article, an approach to employ the electrochemically assisted self-assembly of a multicomponent supramolecular film based on a naturally occurring polyphenol, tannic acid (TA), is featured. Here, the capacity of polyphenolic compounds to form complexes with metal ions, as well as to act both as reducing agents and stabilizers in colloidal synthesis of metal nanoparticles (NPs) is utilized. The electrochemically triggered self-assembly can be coupled with the ion - printing method, in which the targeted metal ion, in this case Al(III), is incorporated into the film during the synthesis and chemically removed afterwards. This procedure results in a template-like structure of the film with openings ready to bind the same metal ion from the probed solution, thus significantly improving the selectivity of the sensor formed and enhancing its applicability for sensing of toxic metal ions in complex aqueous solutions, such as physiological fluids.
\end{abstract}

Keywords: Aluminum sensing · Electrochemical cross-linking · Nanoparticles · Supramolecular film

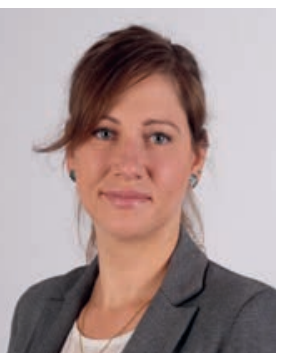

Agata Krywko-Cendrowska obtained her $\mathrm{PhD}$ in chemistry from the University of Warsaw in collaboration with the Department of Physics of the University of Basel as a participant of an International $\mathrm{PhD}$ Program. During her stay in the group of Dr. Boulmedais at Institut Charles Sadron, CNRS, in Strasbourg she worked on electrochemically-assisted self-assembly of supramolecular polyelectrolyte-based thin films for sensing applications. She is currently a postdoctoral researcher at the University of Basel where her focus is on directed insertion of biomolecules into biomimetic membranes. Her research interests are thin functional films and their characterization.

Aluminum is widely used in almost every area of our everyday life, i.e. from food processing, water purification, cosmetics, medicines, vaccinations to even clothing and building construction, due to its incredibly useful physicochemical properties. $\mathrm{Al}$ (III) is an extremely dangerous neurotoxin with poisoning effects (severe headaches, fatigue, loss of memory) that binds to the phosphate groups of nucleic acids influencing their topology, affects gene transcription and accumulates in brain matter. ${ }^{[1-5]}$ Quantification of $\mathrm{Al}(\mathrm{III})$ is crucial especially in the case of children, who are vulnerable due to their low body weight and the exposition to alu- minum via numerous recommended vaccinations, parenteral nutrition or breast milk. ${ }^{[6]} \mathrm{Al}$ (III) levels in patients are conventionally measured using laboratory-based spectroscopic methods ${ }^{[7]}$ such as atomic absorption spectrometry, inductively coupled plasma mass spectrometry or spectrophotometry. Even if these methods offer reasonable sensitivity and reliability, they use expensive instrumentations that require expertise in handling and present the limitation of ex situ analysis. ${ }^{[4]}$ Moreover, the results are not reliable due to the fast metabolism and daily fluctuation in the level of $\mathrm{Al}(\mathrm{III})$ in the human body. ${ }^{[4,7]}$ Routine monitoring of aluminum in biological fluids is thus necessary for biomedical purposes but impossible with the actual methods.

\section{Electrochemical Sensing of Al(III)}

Electrochemical characterization with its high sensitivity, rapid analysis time and cost efficiency, is an excellent technique for the routine monitoring of $\mathrm{Al}(\mathrm{III})$. It is based on a direct tracing of an analyte ion concentration in a solution by monitoring the electrical current at the surface of an electrochemical sensor, which transforms the electrochemical information into an analytically useful signal related to the concentration of the analyte, e.g. current magnitude. Electrochemical sensors and biosensors can offer advantages of low detection limits, a wide linear response range, and good stability and reproducibility via a relatively simple measurement. Although a range of electrochemical, fluorescent or en- 
zyme inhibition-based sensors for $\mathrm{Al}(\mathrm{III})$ have been reported, ${ }^{[8-12]}$ none of these concepts found a practical application because of insufficient sensitivity or precision, indirect measurement, use of mercury electrodes, being sensitive to several interfering species or in most cases not efficient enough to be used in biological fluids. ${ }^{[6,13-20]}$ Recently, two strategies showing promising results based on the inhibition of an enzyme towards $\mathrm{Al}(\mathrm{III})^{[20,21]}$ and on the formation of $\mathrm{Al}(\mathrm{III})$-polyphenol complexes ${ }^{[7]}$ have been introduced. However, none of them could be selectively deposited onto the electrode microarrays as the first one was obtained by manual cross-linking of enzymes and the second one by drop-casting, resulting in weak bonding to the substrate.

\section{Electrochemically-assisted Self-assembly of TA-based Films}

Recently, polyphenols were found to coordinate with metals and self-assemble into combined cross-linked networks of polyphenols and metal ions. ${ }^{[22]}$ The functionality of such networks can be versatile based on the properties of incorporated polyphenols and metal ions. Tannic acid (TA), a natural polyphenolic compound used as an example here, is present in abundance in nature and easily accessible. It is composed of gallic and catecholic moieties, which allow the adhesion on almost any type of the substrate and can interact with atoms or chemical functions in different ways:[23] they can form metal-ligand complexes, charge-transfer complexes, act as a reducing or stabilizing agent to form metal nanoparticles ${ }^{[24]}$ and, when oxidized, covalent intramolecular bonds leading to formation of a film. The formation of the film occurs via cross-linking under oxidative conditions, which can be provided by e.g. a quantum of light or electrical charge. Since TA in its polymeric form is a poor conductor, its progressive deposition onto the electrode upon applying an appropriate potential or current value consecutively decreases the conductivity of the surface. At some point the charge accumulation on the electrode-film interface is observed which stops the electrochemically-driven diffusion of the following TA molecules to the electrode, resulting only in the buildup of thin, electrochemically inactive TA layers (Fig. 1). On the other hand, the ability of TA to form covalent bonds between its molecules under an electrical stimulus, combined with the ability to complex metals like iron, vanadium or aluminum to form nanocoatings ${ }^{[25]}$ can be very well utilized for fabrication of an electrochemical sensor. Here, the incorporated metal ions or nanoparticles will facilitate the electron transport across the film, significantly increasing their conductivity and resulting in electroactive layers.

\subsection{Morphogen-driven Electro Self-assembly}

One recently developed approach to fabricate such electroactive films is electro-triggered self-construction of metal-polyphe- nol films based on a one-pot morphogen driven self-assembly. [24] The presence of catalysts, i.e. morphogens, triggers the film buildup, in the simultaneous presence of all reactants in the solution. ${ }^{[26,27]}$ In analogy with developmental biological processes, a morphogen is defined as a molecule or an ion that is produced at an interface and diffuses into the solution, thus creating a concentration gradient that locally induces a chemical process. ${ }^{[26]}$ The self-construction of metal-polyphenol films is based on the selfassembly of TA with Fe(III) originating from the electro-oxidation of $\mathrm{Fe}$ (II) into $\mathrm{Fe}$ (III) in the presence of TA (Fig 2). First, a solution of TA and $\mathrm{Fe}(\mathrm{II})$, forming a water-soluble ferrous complex, ${ }^{[28]}$ is brought into contact with the working electrode. The application of an anodic current allows the oxidation of $\mathrm{Fe}$ (II) into $\mathrm{Fe}$ (III), forming a confined gradient in the vicinity of the electrode and inducing the localized self-assembly of a TA-Fe(III) film.

Both film thickness and self-assembly kinetics can be tuned by controlling the $\mathrm{Fe}(\mathrm{II}) / \mathrm{TA}$ molar ratio in the building solution, the magnitude, and the duration of the applied current or potential. In contrast to other methods used for the fabrication of TA/Fe(III) layers, ${ }^{[29]}$ the electro-triggered self-assembly allows a localized buildup by electro-generation of $\mathrm{Fe}$ (III) ions on the surface of an electrode and to finely tune the thickness of the coating by switching on and off the electrical stimulus. ${ }^{[26]}$

Although TA remains in a dissociated form in a broad $\mathrm{pH}$ range and the same mass of TA-Fe(III) films can be self-assembled at different $\mathrm{pH}$ values (at $6.25 \mu \mathrm{A} / \mathrm{cm}^{2}$, corresponding to $c a$. $0.3 \mathrm{~V}$ vs. $\mathrm{Ag} / \mathrm{AgCl}$ ), the studies showed that the films assembled at $\mathrm{pH} 7.4$ in comparison to those obtained at $\mathrm{pH} \mathrm{3,} \mathrm{exhibit} \mathrm{a}$ higher stability when brought into contact with a ligand which could strongly bind $\mathrm{Fe}(\mathrm{III})$ ions, EDTA, while tested at the $\mathrm{pH}$ of the buildup. ${ }^{[26]}$

This is consistent with the fact that the number of coordination bonds increases with the $\mathrm{pH}$, leading to more stable TA-Fe(III) complexes. ${ }^{[29]}$ This property is desired, taking into account the applicability of the films for potential sensing of $\mathrm{Al}(\mathrm{III})$ under physiological $\mathrm{pH}$ and in a presence of the $\mathrm{Al}(\mathrm{III})$ ion that forms strong complexes with TA, which could cause the disassembly of the film. Due to the fact that the TA-Al(III) signal is observed at a far anodic potential value $(0.8 \mathrm{~V}$ vs. $\mathrm{Ag} / \mathrm{AgCl})$, this potential value would be sufficient for the TA oxidative cross-linking which occurs in the potential range between 0.4 and $1.1 \mathrm{~V}$ vs. $\mathrm{Ag} / \mathrm{AgCl}$. Therefore, the current magnitude measured during the tracing of $\mathrm{Al}(\mathrm{III})$ in the analyte solution would correspond both to the TA-Al(III) formation and TA oxidative cross-linking. In order to avoid any contribution arising from the oxidation of TA, the deposition was performed at $0.8 \mathrm{~V}$ ensuring the oxidized state of TA molecules and, at the same time, resulting in the significant strengthening of the layers by simultaneous cross-linking of TA. As a result, formation of a
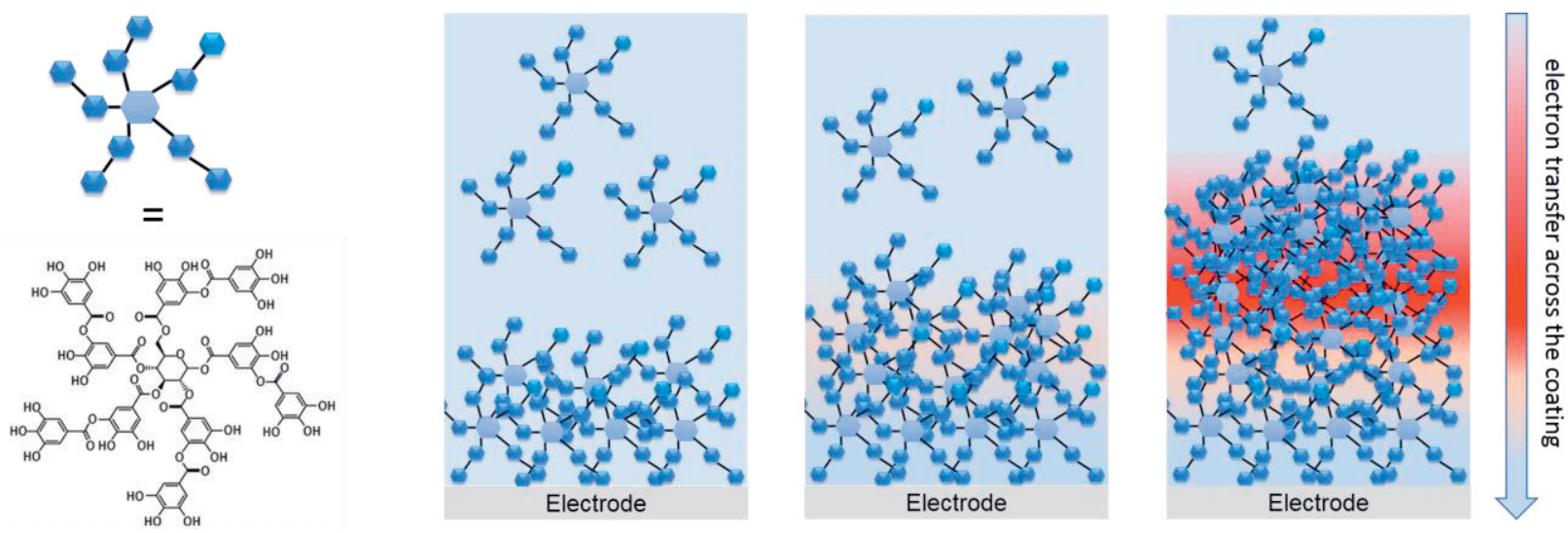

Fig. 1. Schematic representation of the electron transfer across a polymer coating during the buildup. If the polymer formed is nonconductive, the electron transfer is hindered at first and with the increase in film thickness, charge accumulation occurs on the surface of the layer and the electrical charge transport will be blocked. 
a

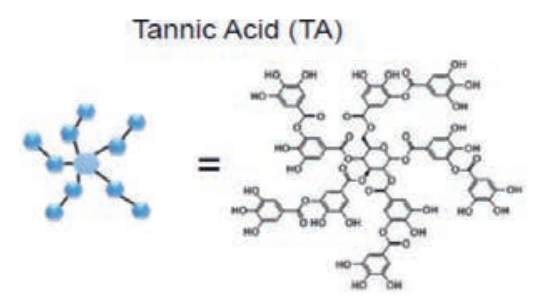

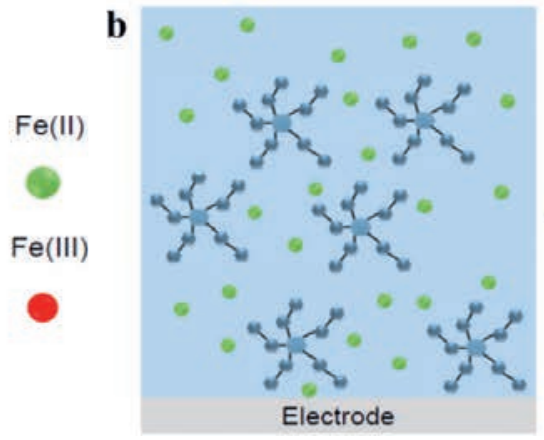

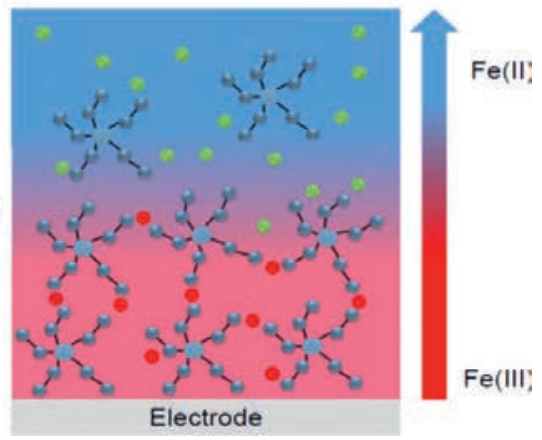

Fig. 2. (a) Chemical structure of tannic acid (TA). (b) Schematic representation of the one-pot self-assembly of a metal-polyphenolic network film based on the electrooxidation of Fe(II) to Fe(III). Copyright (C) 2017, American Chemical Society, ref. [26].

polymeric network with metal ions embedded within and exposed phenolic groups on the surface able to complex Al(III) ions via an electrochemically monitored process was achieved. The mechanism was proven successful both when a semiconductor (ITO ${ }^{[26]}$ ) and a metallic electrode $\left(\mathrm{Au}^{[30]}\right)$ were used as the substrate and could be easily broadened to other conductive solid supports. The procedure was applied for electrochemical tracing of $\mathrm{Al}$ (III) ions with TA-Fe(III) films cross-linked on Au in a $0.15 \mathrm{M} \mathrm{KPF}_{6}$ solution at a physiological $\mathrm{pH}$ level and dependency of the current magnitude on $\mathrm{Al}(\mathrm{III})$ concentration was obtained. ${ }^{[30]}$

\subsection{Electrochemically-assisted Self-assembly of TA-based Films via Ion Printing}

The selectivity of the sensor towards the targeted analyte can be significantly improved if combined with an ion-imprinting approach, which aims to recognize ions while retaining the unique advantages of molecular imprinting technology, namely structure predictability, recognition specificity and universal applicability. ${ }^{[31]}$ In this approach, the targeted metal ion, $\mathrm{Al}(\mathrm{III})$, is incorporated into the film during the self-assembly as TA-Al(III) complexes and chemically removed from the crosslinked TA network afterward with a ligand which can form complexes with Al(III) stronger than TA. The agent typically used for the removal of $\mathrm{Al}(\mathrm{III})$ ions from a polymeric network are fluoride ions, which can form water-soluble and easy to remove complexes of $\mathrm{Al}(\mathrm{III})$ with stability constants higher than those formed with TA by almost two orders of magnitude. As compared to the morphogendriven electroassembly of TA-Fe(III) films, the films would not decompose upon contact with the ligand solution due to the presence of intramolecular TA bonds in the assemblies.

Depending on the targeted solution for the voltammetric tracing of $\mathrm{Al}(\mathrm{III})$, the choice of the substrate should be carefully considered. If the targeted solution is a human physiological fluid, e.g. human serum (HS), the substrate for the deposition should be both chemically and electrochemically stable in the analyzed solution. Due to the fact that HS contains $c a .0 .15 \mathrm{M}$ $\mathrm{NaCl}$, noble metals cannot be used as the substrate (i.e. Au, Ag or $\mathrm{Pt}$ ) because of their instability in the presence of chlorides in an aqueous solution. Although Au exhibited a high applicability as the electrode for electrochemical sensing of $\mathrm{Al}(\mathrm{III})$ in $\mathrm{KPF}_{6}$ solution while decorated with the TA-based sensor film, it dissolves in the presence of chloride ions already at $c a$. $0.6 \mathrm{~V}$ vs. $\mathrm{Ag} / \mathrm{AgCl}$ which is well below the potential at which $\mathrm{Al}(\mathrm{III})$ can be effectively traced. An applicable alternative is to utilize a semiconductive (SC) oxide electrode, e.g. ITO, which is robust in a broad range of electrolytes and has a wide electrochemical window in the anodic range. Typically, the conductivity of SC electrodes is lower than that of metal electrodes, which combined with the poor conductivity of the sensor film results in high detection and quantification limits, as well as lowered sensitivity due to the electron transfer not being efficiently facilitated across the film.
The sensitivity of an electrochemical sensor, on the other hand, can be drastically improved by the incorporation of metal nanoparticles (NPs), e.g. Au or Ag, due to their high electrical conductivity and ability to enhance the electron transfer between the organic film and the electrode. ${ }^{[32-34]}$

Recently, an approach to sensitize the TA-based film in situ with metal nanoparticles proved to enhance its overall electronic properties and the sensitivity towards $\mathrm{Al}$ (III) in a diluted HS solution. ${ }^{[35]}$ Here the assembly of the film was split into two stages: (i) presynthesis of the components and (ii) electrochemically triggered self-assembly of the film onto ITO electrodes (Fig 3). In the first stage, the property of TA as the reducing and stabilizing agent in the colloidal synthesis of metal nanoparticles via a modified procedure was used, resulting in TA-capped NPs sized between 2 and $5 \mathrm{~nm}$. The idea was inspired by ref. [7] where commercially available TA-capped gold nanoparticles drop-casted onto glassy carbon electrode were employed for $\mathrm{Al}(\mathrm{III})$ sensing in an aqueous solution at $\mathrm{pH} 2$. Taking into account the amphoteric character of $\mathrm{Al}$ (III) salts, the proximity of the physiological $\mathrm{pH}$ to the neutral value and a very low concentration of $\mathrm{Al}$ (III) in biological fluids, such approach of a film weakly bonded to the electrode surface would not be efficient enough to be used for aluminum tracing in physiological conditions. Also, while the use of gold, either as an electrode or sensitizer, under physiological conditions is limited due to the electrochemical dissolution in the presence of chlorides, similarly as for Au substrates, AgNPs used here can remain stable in a broad concentration and voltage range ${ }^{[36]}$ in an immobilized form, especially if embedded within a chemically robust network and stabilized by a reducing agent that can control its redox activity, TA. ${ }^{[37]}$ Due to the small difference in the electronegativity of Ag (1.9) and Al (1.5), the authors expected that the addition of $\mathrm{Al}$ (III) into the synthesized nanoparticles would not destabilize the mixture but the TA on the surface of AgNPs would form TA-Al(III) complexes instead. By carefully controlling the TA-Al(III) molar ratio, a sufficient number of TA phenolic groups remained in a dissociated form to undergo the electrochemical cross-linking to form a film.

After applying an electrochemical potential value sufficient to trigger the cross-linking of TA, the Ag-TA-Al(III) could be formed in $\mathrm{KPF}_{6}$ at $\mathrm{pH}$ 7.4. The film was stable both in the basic electrolyte and in the human serum solution diluted to $10 \%$ in volume with $0.15 \mathrm{M} \mathrm{NaCl}$. A linear dependence between the $\mathrm{Al}$ (III) concentration and the current magnitude was measured for the range $2.7-13.3 \mu \mathrm{g} / \mathrm{l}$ of $\mathrm{Al}(\mathrm{III})$, which, if referred to $100 \% \mathrm{HS}$, would encompass the $\mathrm{Al}(\mathrm{III})$ concentration range in healthy individuals to a $\mathrm{Al}(\mathrm{III})$ level considered as clinically toxic.

\section{Conclusions}

An approach based on the electrodeposition of supramolecular metal-polyphenol films for the synthesis of electroactive coatings sensitive to $\mathrm{Al}(\mathrm{III})$ is reviewed. Two novel approaches of an electrochemically triggered self-assembly of thin metal-polyphenol 

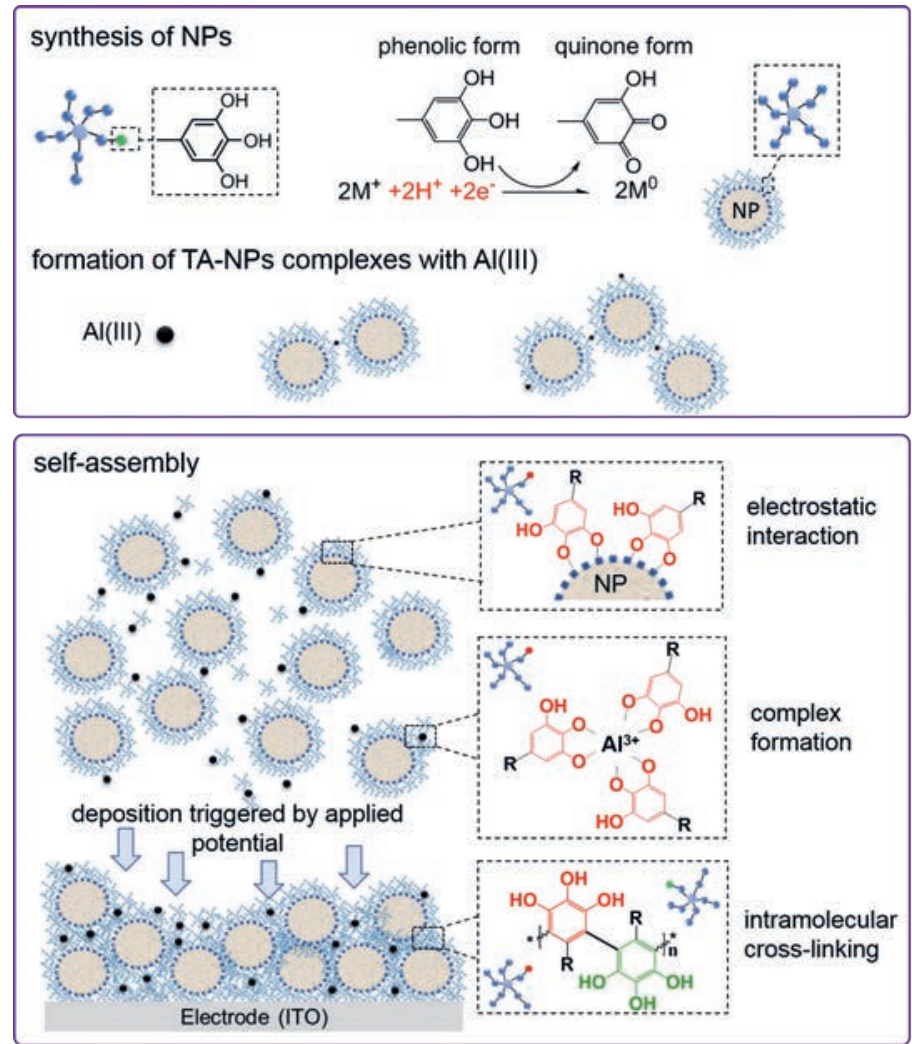

Fig. 3. Schematic presentation of electrochemically-assisted self-assembly of aluminum-imprinted TA-based films sensitized with metal NPs.

Scheme adapted from ref. [35].

films, which could act as sensors for the voltammetric tracing of $\mathrm{Al}(\mathrm{III})$ under physiological conditions, is described. In both cases, the capacity of tannic acid to bind metal ions into watersoluble complexes and to form intramolecular bonds to form thin films were utilized. The applicability of the system obtained via the morphogenic approach was shown in an aqueous electrolyte solution at physiological $\mathrm{pH}$ while the coating obtained via ionprinting and sensitized with metal nanoparticles was successfully employed for the voltammetric tracing of aluminum in human blood serum.

\section{Acknowledgements}

The author acknowledges Idex Post doctoral students and Région Grand Est «Jeunes Chercheures» for financial support. The author would like to thank the Swiss Chemical Society and DSM for the best poster award in the Polymers, Colloids \& Interfaces session, as well as Dr Fouzia Boulmedais and Dr. Laurent Marot for supervision and unlimited support throughout the collaboration.

Received: February 15, 2020
[1] P. Ganrot, Environ. Health Persp. 1986, 65, 363

[2] J. L. Greger, J. E. Sutherland, R. Yokel, Crit. Rev. Clin. Lab. Sci. 1997, 34 , 439.

[3] C. Exley, E. R. House, Monatsh. Chem. 2011, 142, 357.

[4] Y. Liu, Z. Wei, W. Duan, C. Ren, J. Wu, D. Liu, H. Chen, Dyes Pigments 2018, 149, 491.

[5] S. Qiu, Y. Huang, X. He, Z. Sun, P. Liu, C. Liu, Meas. Sci. Techno. 2015, 26, 105101

[6] M. Zeager, A. D. Woolf, R. H. Goldman, Pediatrics 2012, 129, e142.

[7] A. L. Suherman, E. E. Tanner, S. Kuss, S. V. Sokolov, J. Holter, N. P. Young, R. G. Compton, Sensors and Actuators B: Chem. 2018, 265, 682.

[8] F. Zhang, S. Bi, J. Liu, X. Yang, X. Wang, L. Yang, T. Yu, Y. Chen, L. Dai, T. Yang, Anal. Sci. 2002, 18, 293.

[9] M. Arvand, M. Kermanian, M. A. Zanjanchi, Electrochim. Acta 2010, 55, 6946.

[10] V. Arancibia, C. Munoz, Talanta 2007, 73, 546.

[11] L. Qiong, W. Lirong, X. Danli, L. Guanghan, Food Chem. 2006, 97, 176.

[12] J. Di, S. Bi, T. Yang, M. Zhang, Sensors and Actuators B: Chem. 2004, 99 , 468

[13] L. B. Santos, M. T. de Souza, A. T. Paulino, E. E. Garcia, E. M. Nogami, J. C. Garcia, N. E. de Souza, Microchem. J. 2014, 112, 50.

[14] D. Zhang, X. Song, R. Zhang, M. Zhang, F. Liu, Eur. J. Inorg. Chem. 2005 , $2005,1643$.

[15] J. Wang, P. A. Farias, J. S. Mahmoud, Anal. Chim. Acta 1985, 172, 57.

[16] S. D. Thomas, D. E. Davey, D. E. Mulcahy, C. W. Chow, Electroanal. 2006, $18,2257$.

[17] W. P. See, L. Y. Heng, S. Nathan, Anal. Sci. 2015, 31, 997.

[18] S.-C. Chang, J. Sensor Sci. Technol. 2010, 19, 421.

[19] D. V. Vukomanovic, J. A. Page, G. W. Vanloon, Can. J. Chem. 1991, 69, 1418.

[20] M. Barquero, O. Dominguez, M. Alonso, M. Arcos, Chem. Sci. J. 2015, 6, 89.

[21] M. Barquero-Quirós, O. Domínguez-Renedo, M. A. Alonso-Lomillo, M. J. Arcos-Martínez, Sensors 2014, 14, 8203.

[22] X. Zhang, G. Parekh, B. Guo, X. Huang, Y. Dong, W. Han, X. Chen, G. Xiao, Futur. Drug Discov. 2019, 2631.

[23] T. J. Deming, Curr. Opin. Chem. Biol. 1999, 3, 100.

[24] Y. Hao, N. Zhang, J. Luo, X. Liu, Nano 2018, 13, 1850003.

[25] J. Guo, Y. Ping, H. Ejima, K. Alt, M. Meissner, J. J. Richardson, Y. Yan, K. Peter, D. Von Elverfeldt, C. E. Hagemeyer, Angew. Chem. Int. Ed. 2014, 53 , 5546.

[26] C. Maerten, L. Lopez, P. Lupattelli, G. Rydzek, S. Pronkin, P. Schaaf, L. Jierry, F. Boulmedais, Chem. Mater. 2017, 29, 9668.

[27] G. Rydzek, L. Jierry, A. Parat, J. S. Thomann, J. C. Voegel, B. Senger, J. Hemmerlé, A. Ponche, B. Frisch, P. Schaaf, Angew. Chem. Int. Ed. 2011, 50, 4374.

[28] F. Hingston, Soil Res. 1963, 1, 63.

[29] H. Ejima, J. J. Richardson, K. Liang, J. P. Best, M. P. van Koeverden, G. K. Such, J. Cui, F. Caruso, Science 2013, 341, 154.

[30] A. Krywko-Cendrowska, L. Marot, F. Boulmedais, J. Electroanal. Chem., to be published.

[31] J. Fu, L. Chen, J. Li, Z. Zhang, J. Mater. Chem. A 2015, 3, 13598.

[32] X. Luo, A. Morrin, A. J. Killard, M. R. Smyth, Electroanal. 2006, $18,319$.

[33] T. Liu, J. Zhong, X. Gan, C. Fan, G. Li, N. Matsuda, ChemPhysChem 2003, 4, 1364.

[34] X. Gan, T. Liu, J. Zhong, X. Liu, G. Li, ChemBioChem 2004, 5, 1686.

[35] A. Krywko-Cendrowska, L. Marot, F. Boulmedais, ACS Appl. Mater. Interf., to be submitted.

[36] C. m. Levard, S. Mitra, T. Yang, A. D. Jew, A. R. Badireddy, G. V. Lowry, G. E. Brown Jr, Environ. Sci. Technol. 2013, 47, 5738.

[37] A. L. Suherman, G. Zampardi, H. M. Amin, N. P. Young, R. G. Compton, PhysChemChemPhys 2019, 21, 4444.

\section{License and Terms}

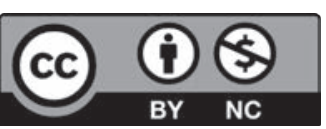

This is an Open Access article under the terms of the Creative Commons Attribution License CC BY_NC 4.0. The material may not be used for commercial purposes.

The license is subject to the CHIMIA terms and conditions: (http:// chimia.ch/component/sppagebuilder/?view=page \&id=12).

The definitive version of this article is the electronic one that can be found at doi:10.2533/chimia.2020.289 